\title{
Improvement of the Determination of the WIMP Mass from Direct Dark Matter Detection Data
}

\author{
Manuel Drees* and Chung-Lin Shan ${ }^{\dagger}$ \\ *Physikalisches Institut der Universität Bonn, D-53115 Bonn, Germany \\ School of Physics, KIAS, Seoul 130-012, Republic of Korea \\ Bethe Center of Theoretical Physics, Universität Bonn, D-53115 Bonn, Germany \\ E-mail: drees@th.physik.uni-bonn.de \\ ${ }^{\dagger}$ School of Physics and Astronomy, Seoul National University, Seoul 151-747, Republic of Korea \\ E-mail: cshan@hep1.snu.ac.kr
}

\begin{abstract}
Weakly Interacting Massive Particles (WIMPs) are one of the leading candidates for Dark Matter. We developed a model-independent method for determining the WIMP mass by using data (i.e., measured recoil energies) of direct detection experiments. Our method is independent of the as yet unknown WIMP density near the Earth, of the form of the WIMP velocity distribution, as well as of the WIMP-nucleus cross section. It requires however positive signals from at least two detectors with different target nuclei. At the first phase of this work we found a systematic deviation of the reconstructed WIMP mass from the real one for heavy WIMPs. Now we improved this method so that this deviation can be strongly reduced for even very high WIMP mass. The statistical error of the reconstructed mass has also been reduced. In a background-free environment, a WIMP mass of $\sim 50 \mathrm{GeV}$ could in principle be determined with an error of $\sim 35 \%$ with only $2 \times 50$ events.
\end{abstract}

Keywords: Dark Matter, direct detection, WIMP mass

PACS: $95.35 .+\mathrm{d}, 29.85 . \mathrm{Fj}$

\section{INTRODUCTION}

By now there is strong evidence that more than $80 \%$ of all matter in the Universe is dark (i.e., interacts at most very weakly with electromagnetic radiation and ordinary matter). The dominant component of this cosmological Dark Matter must be due to some yet to be discovered, non-baryonic particles. Weakly Interacting Massive Particles (WIMPs) $\chi$ with masses roughly between $10 \mathrm{GeV}$ and a few $\mathrm{TeV}$ are one of the leading candidates for Dark Matter (for reviews, see Refs. [1]).

Currently, the most promising method to detect many different WIMP candidates is the direct detection of the recoil energy deposited in a low-background laboratory detector by elastic scattering of ambient WIMPs on the target nuclei [2]. The differential rate for elastic WIMPnucleus scattering is given by [1]:

$$
\frac{d R}{d Q}=\mathscr{A} F^{2}(Q) \int_{v_{\min }}^{\infty}\left[\frac{f_{1}(v)}{v}\right] d v .
$$

Here $R$ is the direct detection event rate, i.e., the number of events per unit time and unit mass of detector material, $Q$ is the energy deposited in the detector, $F(Q)$ is the elastic nuclear form factor, $f_{1}(v)$ is the one-dimensional velocity distribution function of the WIMPs impinging on the detector, $v$ is the absolute value of the WIMP velocity in the laboratory frame. The constant coefficient
$\mathscr{A}$ is defined as

$$
\mathscr{A} \equiv \frac{\rho_{0} \sigma_{0}}{2 m_{\chi} m_{\mathrm{r}, \mathrm{N}}^{2}}
$$

where $\rho_{0}$ is the WIMP density near the Earth and $\sigma_{0}$ is the total cross section ignoring the form factor suppression. The reduced mass $m_{\mathrm{r}, \mathrm{N}}$ is defined by

$$
m_{\mathrm{r}, \mathrm{N}} \equiv \frac{m_{\chi} m_{\mathrm{N}}}{m_{\chi}+m_{\mathrm{N}}}
$$

where $m_{\chi}$ is the WIMP mass and $m_{\mathrm{N}}$ that of the target nucleus. Finally, $v_{\min }=\alpha \sqrt{Q}$ is the minimal incoming velocity of incident WIMPs that can deposit the energy $Q$ in the detector and

$$
\alpha \equiv \sqrt{\frac{m_{\mathrm{N}}}{2 m_{\mathrm{r}, \mathrm{N}}^{2}}} .
$$

In our earlier work [3], we developed methods for reconstructing the one-dimensional velocity distribution, $f_{1}(v)$, and for estimating its moments from the recoil spectrum as well as from measured recoil energies directly in direct detection experiments:

$$
\begin{aligned}
\left\langle v^{n}\right\rangle & \equiv \int_{v_{\min }\left(Q_{\text {thre }}\right)}^{\infty} v^{n} f_{1}(v) d v \\
& =\alpha^{n}\left[\frac{2 Q_{\text {thre }}^{(n+1) / 2} r_{\text {thre }} / F^{2}\left(Q_{\text {thre }}\right)+(n+1) I_{n}}{2 Q_{\text {thre }}^{1 / 2} r_{\text {thre }} / F^{2}\left(Q_{\text {thre }}\right)+I_{0}}\right] . \text { (5) }
\end{aligned}
$$


Here $r_{\text {thre }} \equiv(d R / d Q)_{Q=Q_{\text {thre }}}$ is an estimated value of the scattering spectrum at the threshold energy, $Q_{\text {thre }}$, and $I_{n}$ can be estimated by

$$
I_{n}=\sum_{a} \frac{Q_{a}^{(n-1) / 2}}{F^{2}\left(Q_{a}\right)}
$$

where the sum runs over all events in the data set. Note that Eq.(5) can be used without prior knowledge of the local WIMP density, $\rho_{0}$, of the velocity distribution function of incident WIMPs, $f_{1}(v)$, as well as of the WIMPnucleus cross section, $\sigma_{0}$.

\section{DETERMINING THE WIMP MASS}

By requiring that the values of a given moment of $f_{1}(v)$ estimated by Eq.(5) from two detectors with different target nuclei, $X$ and $Y$, agree, we found a simple expression for determining the WIMP mass [4]:

$$
m_{\chi}=\frac{\sqrt{m_{X} m_{Y}}-m_{X}\left(\mathscr{R}_{n, X} / \mathscr{R}_{n, Y}\right)}{\mathscr{R}_{n, X} / \mathscr{R}_{n, Y}-\sqrt{m_{X} / m_{Y}}}
$$

with

$$
\mathscr{R}_{n, X}=\left[\frac{2 Q_{\mathrm{thre}, X}^{(n+1) / 2} r_{\mathrm{thre}, X} / F_{X}^{2}\left(Q_{\mathrm{thre}, X}\right)+(n+1) I_{n, X}}{2 Q_{\mathrm{thre}, X}^{1 / 2} r_{\mathrm{thre}, X} / F_{X}^{2}\left(Q_{\mathrm{thre}, X}\right)+I_{0, X}}\right]^{1 / n} .
$$

Here $n \neq 0, m_{(X, Y)}$ and $F_{(X, Y)}(Q)$ are the masses and the form factors of the nuclei $X$ and $Y$, respectively, and $r_{\text {thre, }(X, Y)}$ refer to the counting rates at the threshold energies of two detectors. Note that the form factors in the estimates of $I_{n, X}$ and $I_{n, Y}$ using Eq.(6) are different.

Additionally, for the spin-independent (SI) scattering of a supersymmetric neutralino, which is the perhaps best motivated WIMP candidate [1], and for all WIMPs which interact primarily through Higgs exchange, the SI scattering cross section is approximately the same for both protons $\mathrm{p}$ and neutrons $\mathrm{n}$. Writing the "pointlike" cross section $\sigma_{0}$ of Eq.(2) as

$$
\sigma_{0}=\left(\frac{4}{\pi}\right) m_{\mathrm{r}, \mathrm{N}}^{2} A^{2}\left|f_{\mathrm{p}}\right|^{2},
$$

where $f_{\mathrm{p}}$ is the effective $\chi \chi \mathrm{pp}$ four-point coupling, $A$ is the atomic number of the target nucleus, one finds

$$
\rho_{0}\left|f_{\mathrm{p}}\right|^{2}=\frac{\pi}{4 \sqrt{2}}\left(\frac{m_{\chi}+m_{\mathrm{N}}}{\mathscr{E} A^{2} \sqrt{m_{\mathrm{N}}}}\right)\left[\frac{2 Q_{\mathrm{thre}}^{1 / 2} r_{\text {thre }}}{F^{2}\left(Q_{\text {thre }}\right)}+I_{0}\right] .
$$

The factor $\mathscr{E}$ appearing in the denominator is the exposure of the experiment, which is dimensionless in natural units; it relates the actual counting rate to the normalized rate (1). Since the unknown factor $\rho_{0}\left|f_{\mathrm{p}}\right|^{2}$ on the lefthand side here is identical for different targets, Eq.(10) leads to another expression for determining $m_{\chi}$ :

$$
m_{\chi}=\frac{\left(m_{X} / m_{Y}\right)^{5 / 2} m_{Y}-m_{X}\left(\mathscr{R}_{\sigma, X} / \mathscr{R}_{\sigma, Y}\right)}{\mathscr{R}_{\sigma, X} / \mathscr{R}_{\sigma, Y}-\left(m_{X} / m_{Y}\right)^{5 / 2}} .
$$

Here we have assumed $m_{(X, Y)} \propto A_{(X, Y)}$, and introduced the quantity

$$
\mathscr{R}_{\sigma, X}=\frac{1}{\mathscr{E}_{X}}\left[\frac{2 Q_{\text {thre }, X}^{1 / 2} r_{\text {thre }, X}}{F_{X}^{2}\left(Q_{\text {thre }, X}\right)}+I_{0, X}\right] .
$$

In order to yield the best-fit value of $m_{\chi}$ as well as its statistical error, we introduced a $\chi^{2}$ function:

$$
\chi^{2}=\sum_{i, j}\left(f_{i, X}-f_{i, Y}\right) \mathscr{C}_{i j}^{-1}\left(f_{j, X}-f_{j, Y}\right) .
$$

It combines the estimators for different $n$ in Eq.(7) with each other, and with the estimator in Eq.(11). In Eq.(13) we defined

$$
f_{i, X}=\left(\frac{\alpha_{X} \mathscr{R}_{i, X}}{300 \mathrm{~km} / \mathrm{s}}\right)^{i},
$$

for $i=-1,1,2, \ldots, n_{\max } ;$ and

$$
f_{n_{\max }+1, X}=\frac{A_{X}^{2}}{\mathscr{R}_{\sigma, X}}\left(\frac{\sqrt{m_{X}}}{m_{\chi}+m_{X}}\right) ;
$$

we analogously defined also $n_{\max }+2$ functions $f_{i, Y}$. Here $n_{\max }$ determines the highest moment of $f_{1}(v)$ that is included in the fit. The $f_{i}$ are normalized such that they are dimensionless and very roughly of order unity; this alleviates numerical problems associated with the inversion of their covariance matrix. The first $n_{\max }+1$ functions $f_{i}$ are basically our estimators of the moments in Eq.(5). The last function is essentially the right-hand side in Eq.(10). It is important to note that $m_{\chi}$ in Eqs.(14a) and (14b) is now a fit parameter, not the true (input) value of the WIMP mass. Recall also that our estimator (6) for $I_{n}$ appearing in Eqs.(14a) and (14b) is independent of $m_{\chi}$. Hence the first $n_{\max }+1$ fit functions depend on $m_{\chi}$ only through the overall factor $\alpha^{i}$.

Moreover, $\mathscr{C}$ here is the total covariance matrix. Since the $X$ and $Y$ quantities are statistically completely independent, $\mathscr{C}$ can be written as a sum of two terms:

$$
\mathscr{C}_{i j}=\operatorname{cov}\left(f_{i, X}, f_{j, X}\right)+\operatorname{cov}\left(f_{i, Y}, f_{j, Y}\right) .
$$

The entries of this matrix involving only the moments of the WIMP velocity distribution can be read off Eq.(82) of Ref. [3], with an obvious modification due to the normalization factor in Eq.(14a). Since the last $f_{i}$ defined in Eq.(14b) can be computed from the same basic quantities, i.e., the counting rates at $Q_{\text {thre }}$ and the integrals $I_{0}$, the entries of the covariance matrix involving this last fit function can also be computed straightforwardly. 


\section{MATCHING THE CUT-OFF ENERGIES}

The basic requirement of our method for determining $m_{\chi}$ given in Eq.(7) is that, from two experiments with different target nuclei, the values of a given moment of the WIMP velocity distribution estimated by Eq.(5) should agree. This means that the upper cuts on $f_{1}(v)$ in two data sets should be (approximately) equal. ${ }^{1}$ Since $v_{\text {cut }}=\alpha \sqrt{Q_{\max }}$, it requires that

$$
Q_{\max , \mathrm{Y}}=\left(\frac{\alpha_{X}}{\alpha_{Y}}\right)^{2} Q_{\max , \mathrm{X}} .
$$

Note that $\alpha$ defined in Eq.(4) is a function of the true WIMP mass. Thus this relation for matching optimal cut-off energies can be used only if $m_{\chi}$ is already known. One possibility to overcome this problem is to fix the cut-off energy of the experiment with the heavier target, minimize the $\chi^{2}\left(m_{\chi, \text { rec }}\right)$ function defined in Eq.(13), and estimate the cut-off energy for the lighter nucleus by Eq.(16) algorithmically.

In Figs. 1 we show our numerical results for the reconstructed WIMP mass based on Monte Carlo simulations. ${ }^{76} \mathrm{Ge}$ and ${ }^{28} \mathrm{Si}$ have been chosen as two targets. The dotted (green) lines show results for $Q_{\max , \mathrm{Ge}}=$ $Q_{\mathrm{max}, \mathrm{Si}}=100 \mathrm{keV}$ with a systematic deviation discussed in Ref. [4], whereas the solid (black) lines have been obtained by using Eq.(16) with $Q_{\max , \mathrm{Ge}}=100 \mathrm{keV}$ and the true (input) WIMP mass $m_{\chi}$,in. The dashed (red) lines are for the case that $Q_{\text {max }, \mathrm{Ge}}=100 \mathrm{keV}$, whereas $Q_{\max , \mathrm{Si}}$ has been chosen such that $\chi^{2}\left(m_{\chi, \text { rec }}\right)$ is minimal. As shown here, with only 50 events (upper frame) from one experiment, our algorithmic process seems already to work pretty well for WIMP masses up to $\sim 500 \mathrm{GeV}$.

\section{SUMMARY}

In this paper we described the basic ideas of our method for determining the WIMP mass and gave the main formulae. The algorithmic process for correcting the systematic deviation of the reconstructed WIMP mass has also been discussed. More details and discussions about determining $m_{\chi}$ can be found in Ref. [6].

\section{ACKNOWLEDGMENTS}

This work was partially supported by the Marie Curie Training Research Network "UniverseNet" under contract no. MRTN-CT-2006-035863 as well as by the European Network of Theoretical Astroparticle Physics

\footnotetext{
${ }^{1}$ Here we assume the threshold energies to be negligibly small.
}
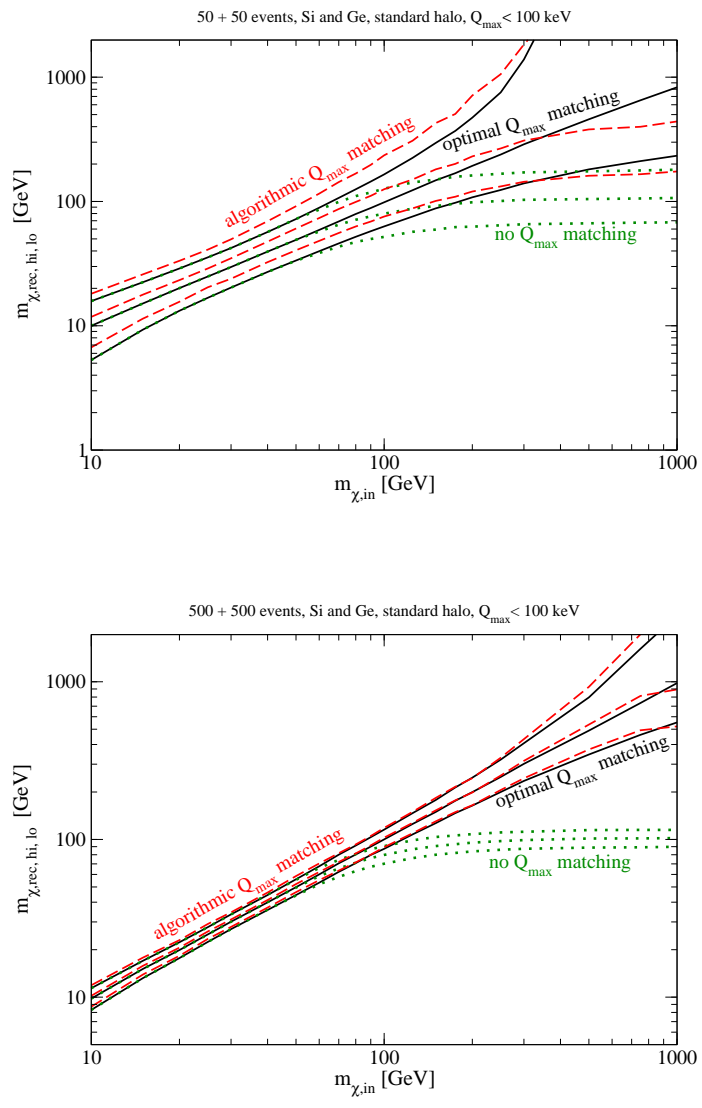

FIGURE 1. Results for the reconstructed WIMP mass as well as its error interval based on the combined fit, using Eq.(13) with $n_{\max }=2$. We assume that the scattering cross section is dominated by spin-independent interactions, The theoretical predicted recoil spectrum for the shifted Maxwellian velocity distribution function [1], [3] with Woods-Saxon elastic form factor [5], [1] $\left(v_{0}=220 \mathrm{~km} / \mathrm{s}, v_{e}=231 \mathrm{~km} / \mathrm{s}\right)$ have been used. We simulated with 50 (upper) and 500 (lower) events on average from each experiment before cuts.

ENTApP ILIAS/N6 under contract no. RII3-CT-2004506222.

\section{REFERENCES}

1. G. Jungman, M. Kamionkowski, and K. Griest, Phys. Rep. 267, 195 (1996); G. Bertone, D. Hooper, and J. Silk, Phys. Rep. 405, 279 (2005).

2. P. F. Smith and J. D. Lewin, Phys. Rep. 187, 203 (1990);

J. D. Lewin and P. F. Smith, Astropart. Phys. 6, 87 (1996).

3. M. Drees and C. L. Shan, JCAP 0706, 011 (2007).

4. C. L. Shan and M. Drees, proceedings of SUSY07 (2007), arXiv:0710.4296 [hep-ph].

5. J. Engel, Phys. Lett. B 264, 114 (1991).

6. M. Drees and C. L. Shan, JCAP 0806, 012 (2008). 